\title{
Nonlinear Breit-Wheeler process in short laser double pulses
}

\author{
A. I. Titov \\ Bogoliubov Laboratory of Theoretical Physics, JINR, Dubna 141980, Russia \\ H. Takabe \\ Helmholtz-Zentrum Dresden-Rossendorf, 01314 Dresden, Germany \\ B. Kämpfer \\ Helmholtz-Zentrum Dresden-Rossendorf, 01314 Dresden, Germany \\ and Institut für Theoretische Physik, TU Dresden, 01062 Dresden, Germany
}

(Received 3 July 2018; published 29 August 2018)

\begin{abstract}
The nonlinear (strong-field) Breit-Wheeler $e^{+} e^{-}$pair production by a probe photon traversing two consecutive short and ultrashort (subcycle) laser pulses is considered within a QED framework. The temporal shape of the pulses and the distance between them are essential for the differential cross section as a function of the azimuthal angle distribution of the outgoing electron (positron). The found effect of a pronounced azimuthal anisotropy is important for subcycle pulses and decreases rapidly with increasing width of the individual pulses.
\end{abstract}

DOI: $10.1103 /$ PhysRevD.98.036022

\section{INTRODUCTION}

The rapidly progressing laser technology [1] offers novel and unprecedented opportunities to investigate quantum systems with intense laser beams [2]. An intensity $I_{L}$ of $\sim 2 \times 10^{22} \mathrm{~W} / \mathrm{cm}^{2}$ has been already achieved [3]. Intensities of the order of $I_{L} \sim 10^{23}-10^{25} \mathrm{~W} / \mathrm{cm}^{2}$ are envisaged in the near future, e.g., at CLF [4], ELI [5], or HiPER [6]. Further facilities are in the planning or construction stages, e.g., the PEARL laser facility [7] at Sarov/Nizhny Novgorod, Russia. The high intensities are provided in short pulses on a femtosecond pulse duration level $[2,8,9]$, with only a few oscillations of the electromagnetic field or even sub-cycle pulses. (The tight connection of high intensity and short pulse duration is further emphasized in [10]. The attosecond regime will become accessible at shorter wavelengths $[11,12])$.

New laser facilities may utilize short and ultrashort pulses in "one-" or "few-" cycle regimes. In this case, a determination of the pulse fine-structure is very important and, in particular, tasking the phase difference between the electric field and pulse envelope, i. e. the carrier envelope phase (CEP). It was found that the CEP effect is especially important just for the case of the short and ultrashort

Published by the American Physical Society under the terms of the Creative Commons Attribution 4.0 International license. Further distribution of this work must maintain attribution to the author(s) and the published article's title, journal citation, and DOI. (subcycle) pulses (cf. [13,14] and references therein; for recent access option for the CEP in long pulses, cf. [15]).

The study of quantum processes in two consecutive (or double) laser pulses with taking into account CEP effects is a new important and interesting topic in laser physics. An analysis of Breit-Wheeler pair production within the framework of scalar electrodynamics is provided in [16]. Below, we give a further development of this problem. We extend that previous consideration to $\mathrm{QED}$, where $e^{+} e^{-}$are fermions, and we concentrate our attention on the azimuthal angle distribution of the outgoing electron (positron) at fixed CEP. In fact, the nonlinear Breit-Wheeler process considered below, assumes the interaction of a probe photon $X$ (e.g., from Compton backscattering in a prepulse, starting a seeded cascade via two-step part of trident process, cf. $[17,18]$ ) with two consecutive laser pulses, $L_{1+2}$, in the reaction $X+L_{1+2} \rightarrow e^{+}+e^{-}$, where a multitude of laser photons can participate simultaneously in the $e^{+} e^{-}$pair creation, thus enabling the process even "below threshold." In the Furry picture, this process is a cross channel of the nonlinear Compton process without having a classical counterpart, as the Thomson process in the weakfield regime. The nonlinear Breit-Wheeler process means the decay of the probe photon into a laser-dressed electron $\left(e^{-}\right)$positron $\left(e^{+}\right)$pair. The emphasis here is on short and intense laser pulses. Long and weak laser pulses are dealt with in the standard textbook Breit-Wheeler process (cf. the review paper [19]). Various aspects, such as the impact of the pulse envelope, pulse duration, and pulse polarization of $e^{+} e^{-}$pair creation in single pulses were analyzed, e.g., in 
Refs. [20-24] with special emphasis on CEP effects [13,14], spin effects [25], bi-frequent pulses [26-28], spectral caustics [27], and focusing effects [29,30].

One may contrast the single-pulse laser beams to a long train of pulses or multipulses, where a special modulation of the phase space distribution of produced $e^{ \pm}$in comb structures arises due to an interference effect [31]. In some sense, such a situation refers to multishot experiments, where a laser with extremely high repetition rate fires for some time. (Another option is the pulse train generation at XFELs, cf. [32].) In between is the presently considered case of a double pulse. Below, we concentrate mainly on the interplay of the effect of CEP and the shape of the laser beams which is determined by the temporal shape of the individual pulses (cf. [22]) and the separation distance between them.

Our present paper is a follow-up of [14] which is inspired by [16]. Differences are (i) Fermion pairs ([16] deals with Boson pairs), (ii) circular polarization ([16] uses linear polarization), (iii) many details of the laser pulse modeling ([16] uses separate individual pulses with particular envelope functions, while our field arises from "cutting out" the pulses by a double-hump window function with hump separation $\geq 0$ ), (iv) consideration of very short and subcycle pulses, (v) focus on subthreshold pair production, and (vi) focus on azimuthal angle distributions ([16] considers the energy spectra).

Our paper is organized as follows. In Sec. II, the doublepulse field model is presented. Section III recalls the basic expressions for the relevant observables in nonlinear BreitWheeler $e^{+} e^{-}$pair production with details relegated to the Appendix. In Sec. IV we discuss results of numerical calculations. Our summary is given in Sec. V.

\section{MODEL OF THE DOUBLE PULSE}

In the following we use the electromagnetic (e.m.) fourpotential for a circularly polarized laser field in the axial gauge $A^{\mu}=(0, \mathbf{A}(\phi))$ with

$$
\mathbf{A}(\phi)=f(\phi)\left(\mathbf{a}_{1} \cos (\phi+\tilde{\phi})+\mathbf{a}_{2} \sin (\phi+\tilde{\phi})\right),
$$

where $\tilde{\phi}$ is the CEP. The quantity $\phi=k \cdot x$ is the invariant phase with four-wave vector $k=(\omega, \mathbf{k})$, obeying the null field property $k^{2}=k \cdot k=0^{1}$ (a dot between four-vectors indicates the Lorentz scalar product) implying $\omega=|\mathbf{k}|$, $\mathbf{a}_{(1,2)} \equiv \mathbf{a}_{(x, y)} ;\left|\mathbf{a}_{x}\right|^{2}=\left|\mathbf{a}_{y}\right|^{2}=a^{2}, \mathbf{a}_{x} \mathbf{a}_{y}=0$; transversality means $\mathbf{k} \mathbf{a}_{x, y}=0$ in the present gauge. The envelope function $f(\phi)$ in case of two consecutive pulses is chosen as sum of two hyperbolic secants

\footnotetext{
${ }^{1}$ Effects of an ambient medium, e.g., a plasma, can be accommodated in a modified dispersion relation, $k^{2} \neq 0$ [33], as customary done in many phenomenological QCD approaches, e.g., in [34].
}

$$
f(\phi)=\frac{1}{\cosh \left(\frac{\phi}{\Delta}-G\right)}+\frac{1}{\cosh \left(\frac{\phi}{\Delta}+G\right)},
$$

where $G$ is the phase separation parameter of two consecutive short pulses at $\pm G \Delta$. Accordingly, the distance between the centers of the two consecutive pulses amounts $2 \pi G N=2 G \Delta$. The dimensionless quantity $\Delta$ is related to the single pulse duration $2 \Delta=2 \pi N$, where $N$ has the meaning of a number of cycles in the individual pulse. It is related to the time duration of the pulse $\tau=2 N / \omega$.

One may imagine the generation of the above described pulse structures by the sequence of a laser beam-beam splitter - a delay section for one of the split beams and subsequently the merging of both beams. Presently, the alignment of two separate laser beams of seems hardly possible to be realized with equal carrier envelope phases and precisely adjustable temporal pulse delay together with keeping constant the polarizations. In this respect, our field model of Eqs. (1) and (2) is highly idealized, but enjoys a minimum number of parameters. ${ }^{2}$ However, having in mind principle effects of quantum interference patterns by such double-slit phenomena in the temporal domain, our field model can be regarded as useful representative ansatz. For a further discussion of aspects of double-and multislit phenomena and interference effects we refer the interested reader to $[16,26]$ with references quoted therein.

For an illustration, in Fig. 1 we show the $x$ component of the e.m. potential $A_{x} / a$ for a subcycle pulse as a function of invariant phase $\phi$ for different values of the separation parameter $G$. The case $G=0$ corresponds to the complete overlap of the two envelopes which leads to the e.m. potential with a doubled amplitude $a$. The e.m. potential $A_{x} / a$ for a short pulse with $N=2$ and different values of $G$ is exhibited in Fig. 2.

As mentioned above, the interplay between carrier envelope phase $\tilde{\phi}$ and separation parameter $G$ is the main subject of our present discussion and, as we will show, it has a strong impact on the azimuthal angle distribution of the outgoing electron (positron), in particular for a very short pulse duration $\Delta$. We will consider essentially multiphoton events, where a finite number of laser photons is involved in the $e^{+} e^{-}$pair production. This allows for the subthreshold $e^{+} e^{-}$pair production with $s<s_{\mathrm{thr}}$, where $s=\left(k_{X}+k\right)^{2}$ is the square of total energy in the center of mass system (c.m.s., defined by $\mathbf{k}_{X}=-\mathbf{k}$, $s_{\text {c.m.s. }}=4 \omega_{X} \omega$ ), $s_{\text {thr }}=4 m^{2}$ is it threshold value, where $m$ is the electron mass. We also discuss the dependence of the cross section on the e.m. field intensity which is described by the reduced field intensity parameter $\xi^{2}=e^{2} a^{2} / \mathrm{m}^{2}$. We use natural units with $c=\hbar=1, e^{2} / 4 \pi=\alpha \approx 1 / 137.036$.

\footnotetext{
${ }^{2}$ Additional parameters are needed for nonidentical pulses, e.g., facilitated by different CEPs, frequencies and field strengths, for instance; cf. [16] for a suitable model and implications for Boson pairs.
} 

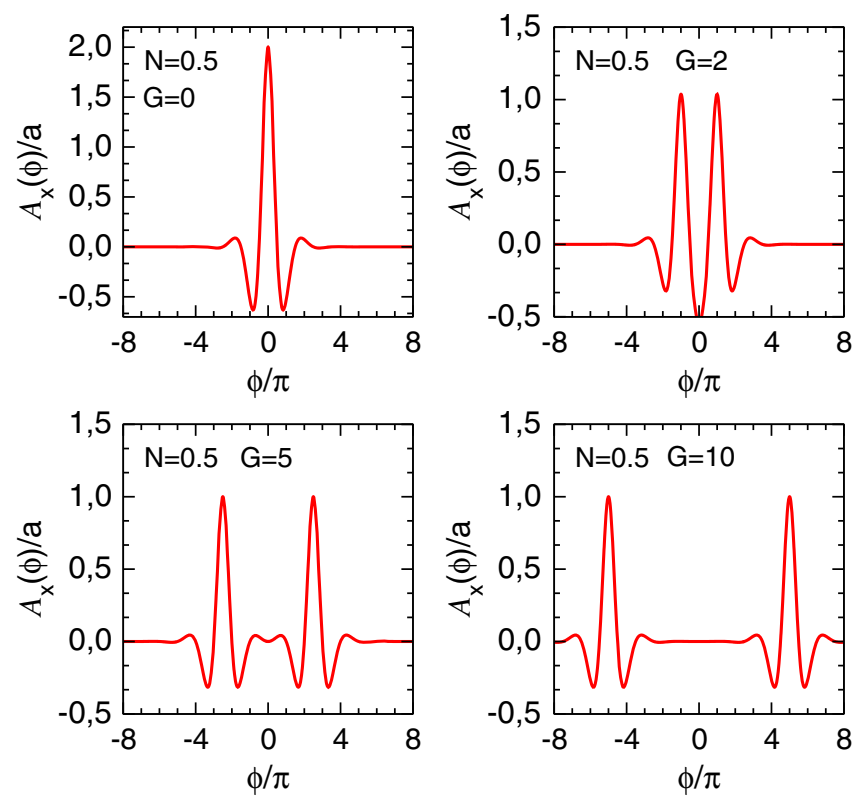

FIG. 1. The e.m. potential $A_{x} / a$ in Eqs. (1), (2) as a function of the invariant phase $\phi=k \cdot x$ for subcycle pulse with $N=1 / 2$ and different values of the separation parameter $G=0,2,5,10$ as it is indicated in the legends.
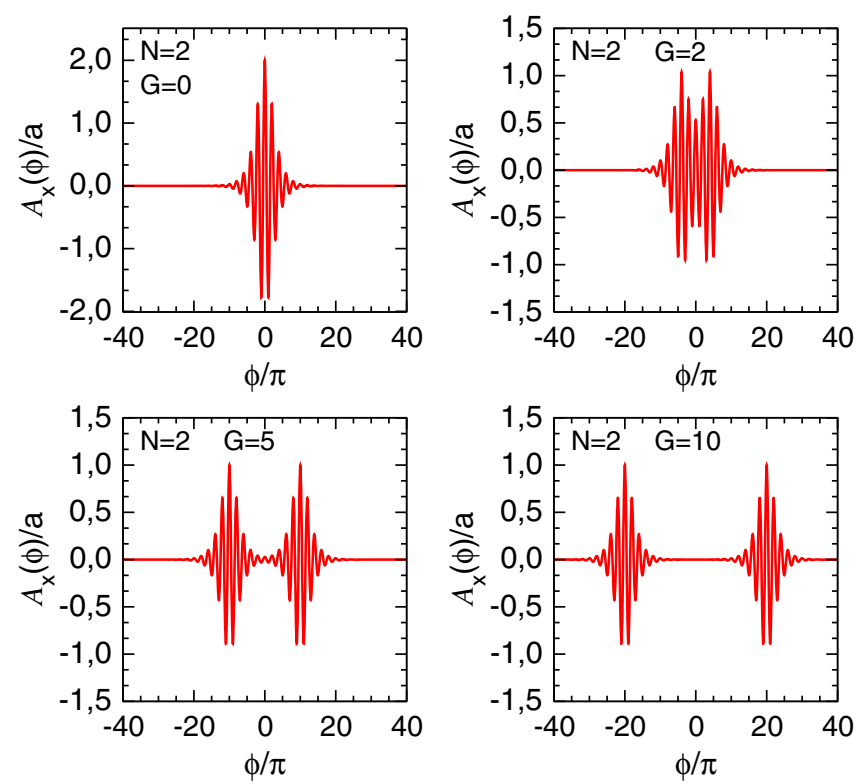

FIG. 2. The same as in Fig. 1 but for a short pulse with $N=2$.

\section{CROSS SECTION AND ANISOTROPY}

The azimuthal angular-differential cross can be cast into the form (cf. Appendix for details)

$$
\frac{d \sigma}{d \phi_{e^{+}}}=\frac{\alpha^{2} \zeta}{4 m^{2} \xi^{2} N_{0}} \int_{\zeta}^{\infty} d \ell v(\ell) \int_{-1}^{1} d \cos \theta_{e^{+}} w(\ell)
$$

with partial probabilities

$$
\begin{aligned}
w(\ell)= & 2\left|\tilde{Y}_{\ell}(z)\right|^{2}+\xi^{2}(2 u-1) \\
& \times\left(\left|Y_{\ell-1}(z)\right|^{2}+\left|Y_{\ell+1}(z)\right|^{2}-2 \operatorname{Re}\left(\tilde{Y}_{\ell}(z) X_{\ell}^{*}(z)\right)\right),
\end{aligned}
$$

which recover the known expressions [19] in case of infinitely long e.m. pulses, where $\ell$ becomes a discrete (integer) variable (cf. [14]). The azimuthal angle of the outgoing positron, $\phi_{e^{+}}$, is defined as $\cos \phi_{e^{+}}=\mathbf{a}_{x} \mathbf{p}_{e^{+}} /$ $a\left|\mathbf{p}_{e^{+}}\right|$. It is related to the azimuthal angle of the electron by $\phi_{e^{-}}=\phi_{e^{+}}-\pi$. Furthermore, $\theta_{e^{+}}$is the polar angle of the outgoing positron, $v$ is the positron (electron) velocity in c.m.s.. The averaging and sum over the spin variables in the initial and the final states is executed.

The lower limit of the integral over the variable $\ell$ is the threshold parameter $\zeta=s_{\mathrm{thr}} / \mathrm{s} \equiv 4 \mathrm{~m}^{2} / \mathrm{s}$. The region of $\zeta<1$ corresponds to the above-threshold $e^{+} e^{-}$pair production, while the region of $\zeta>1$ matches the subthreshold pair production. We keep our notation of [14] and denote fourvectors $k(\omega, \mathbf{k}), \quad k^{\prime}\left(\omega^{\prime}, \mathbf{k}^{\prime}\right) \equiv k_{X}\left(\omega_{X}, \mathbf{k}_{X}\right), \quad p(E, \mathbf{p})$ and $p^{\prime}\left(E^{\prime}, \mathbf{p}^{\prime}\right)$ as the four-momenta of the background (laser) field (1), incoming probe photon, outgoing positron and electron, respectively. The variables $s, v$ and $u$ are determined by $s=2 k \cdot k^{\prime}=2\left(\omega^{\prime} \omega-\mathbf{k}^{\prime} \mathbf{k}\right)$ (with $\mathbf{k}^{\prime} \mathbf{k}=-\omega^{\prime} \omega$ for head-on geometry), $v^{2}=\left(\ell s-4 m^{2}\right) / \ell s, u \equiv\left(k^{\prime} \cdot k\right)^{2} /$ $\left(4(k \cdot p)\left(k \cdot p^{\prime}\right)\right)=1 /\left(1-v^{2} \cos ^{2} \theta_{e}\right)$. The factor $N_{0}$ reads $N_{0}=1 / 2 \pi \int_{-\infty}^{\infty} d \phi\left(f^{2}(\phi)+f^{\prime 2}(\phi)\right)$ and normalizes to the photon flux in case of finite pulses [35]. The variable $\ell$ takes continuous values and the product $\ell \omega$ has the meaning of the laser energy involved in the process (see also [13] for a recent discussion).

The basic functions $Y_{\ell}$ and $X_{\ell}$ entering partial probabilities (4) may be considered as generalized Bessel functions for the finite e.m. pulse,

$$
\begin{gathered}
Y_{\ell}(z)=\frac{1}{2 \pi} \mathrm{e}^{-i \ell\left(\phi_{0}-\tilde{\phi}\right)} \int_{-\infty}^{\infty} d \phi f(\phi) \mathrm{e}^{i \ell \phi-i \mathcal{P}(\phi)}, \\
X_{\ell}(z)=\frac{1}{2 \pi} \mathrm{e}^{-i \ell\left(\phi_{0}-\tilde{\phi}\right)} \int_{-\infty}^{\infty} d \phi f^{2}(\phi) \mathrm{e}^{i \ell \phi-i \mathcal{P}(\phi)}, \\
\tilde{Y}_{\ell}(z)=\frac{z}{2 \ell}\left(Y_{\ell+1}(z)+Y_{\ell-1}(z)\right)-\xi^{2} \frac{u}{u_{\ell}} X_{\ell}(z)
\end{gathered}
$$

with the phase function

$$
\begin{aligned}
\mathcal{P}(\phi)= & z \int_{-\infty}^{\phi} d \phi^{\prime} \cos \left(\phi^{\prime}-\phi_{0}+\tilde{\phi}\right) f\left(\phi^{\prime}\right) \\
& -\xi^{2} \zeta u \int_{-\infty}^{\phi} d \phi^{\prime} f^{2}\left(\phi^{\prime}\right),
\end{aligned}
$$

where $\phi_{0}$ is the azimuthal angle of the outgoing electron $\phi_{0} \equiv \phi_{e^{-}}=\phi_{e+}-\pi$, and the argument $z$ of the generalized Bessel functions is related to $\xi, \ell$ and $u$ via $z=$ $2 \ell \xi \sqrt{u / u_{\ell}\left(1-u / u_{\ell}\right)}$ with $u_{\ell} \equiv \ell / \zeta$. 
Together with the differential cross section $d \sigma / d \phi_{e^{+}}$ we analyze the anisotropy as a function of the positron azimuthal angle $\phi_{e^{+}}$(we do not need to fix explicitly azimuthal angle $\phi_{e^{-}}$of the electron because of the above relation $\left.\phi_{e^{+}}=\phi_{e^{-}}+\pi\right)$

$$
\mathcal{A} \equiv \mathcal{A}\left(\phi_{e^{+}}\right)=\frac{d \sigma /\left.d \phi_{e^{+}}\right|_{\phi_{e^{+}}}-d \sigma /\left.d \phi_{e^{+}}\right|_{\phi_{e^{+}+\pi}}}{d \sigma /\left.d \phi_{e^{+}}\right|_{\phi_{e^{+}}}+d \sigma /\left.d \phi_{e^{+}}\right|_{\phi_{e^{+}+\pi}}} .
$$

The cross section (3) and anisotropy (9) have a nontrivial, nonmonotonic dependence as a function of the azimuthal angle $\phi_{e^{+}}$(or $\phi_{e^{-}}$), which is determined by the values of the carrier phase $\tilde{\phi}$ and separation parameter $G$. On a qualitative level, the reason for such behavior is the following. The basic functions $Y_{\ell}$ and $X_{\ell}$ are determined by the integral over $d \phi$ with a rapidly oscillating exponential function $\exp [i \Psi]$ with leading terms

$$
\begin{aligned}
\Psi= & \ell \phi-z \Delta \cos (\Phi-G \Delta) \int_{-\infty}^{\frac{\phi}{\Delta}-G} d t f_{0}(t) \cos (t \Delta) \\
& -z \Delta \sin (\Phi-G \Delta) \int_{-\infty}^{\frac{\phi}{\Delta}-G} d t f_{0}(t) \sin (t \Delta) \\
& -z \Delta \cos (\Phi+G \Delta) \int_{-\infty}^{\frac{\phi}{\Delta}+G} d t f_{0}(t) \cos (t \Delta) \\
& -z \Delta \sin (\Phi+G \Delta) \int_{-\infty}^{\frac{\phi}{\Delta}+G} d t f_{0}(t) \sin (t \Delta), \\
& +\cdots,
\end{aligned}
$$

where $f_{0}(t)=1 / \cosh (t)$ and $\Phi=\phi_{e^{-}}-\tilde{\phi}$. The ellipses refer to contributions from the second line in (8). Due to the fact that for short (ultrashort) pulses

$$
\int_{-\infty}^{t} d t^{\prime} f_{0}\left(t^{\prime}\right) \cos \left(t^{\prime} \Delta\right) \gg \int_{-\infty}^{t} d t^{\prime} f_{0}\left(t^{\prime}\right) \sin \left(t^{\prime} \Delta\right)
$$

one can conclude that the maximum value to the highly oscillating integrals, determined by the basic functions $Y_{\ell}$ and $X_{\ell}$, comes from the range $\Phi \pm G \Delta \simeq 2 n \pi$, where $n$ is an integer $n=0,1,2 \ldots$. Then the differential cross section would be enhanced at

$$
\phi_{e^{+}}^{\max }-\tilde{\phi}=(2 n+1) \pi \pm G \Delta .
$$

\section{NUMERICAL RESULTS}

\section{A. Azimuthal distributions}

Let us consider first the subcycle pulse with $N=1 / 2$, i.e., $\Delta=\pi / 2$. For subcycle pulses, the electric field vector does not rotate in the $\mathbf{k}$-transverse plane with full length. This asymmetry, which is also seen in Fig. 1 as strong asymmetry in the $\pm A_{x}$ directions, leaves such a strong imprint on the azimuthal distribution of $e^{ \pm}$. It rapidly disappears for longer pulses, i.e., larger values of $N$, as already evidenced by Fig. 2.

To be specific we consider the differential cross section $d \sigma / d \phi_{e^{+}}$for subcycle pulse with $N=1 / 2$ as a function of azimuthal angle of positron momentum at different values of separation parameter $G$ and fixed CEP $\tilde{\phi}=0$ exhibited in the left panel of Fig. 3. One can see the oscillating structure of the cross section. The positions of the maxima and the frequencies of the oscillations depend on the separation parameter.

The dependence of the differential cross section for ultrashort pulses on CEP at fixed separation parameter $G=8$ is exhibited in the right panel of Fig. 3. One can see that the cross sections have a bumplike structure and, besides, the bump positions coincide with the corresponding carrier phases. If one plots the cross sections as a function of the useful "scale variable"

$$
\chi_{e^{+}}=\phi_{e^{+}}-\tilde{\phi}
$$

then all curves displayed in the right panel of Fig. 3 merge into one (that is the blue one labeled by $\tilde{\phi}=0$ ). The entire dependence of the differential cross section on $\tilde{\phi}$ is contained in the variable $\chi_{e^{+}}$(cf. [14]). We use this variable in our subsequent discussion.

According to (12) the differential cross section would be enhanced at

$$
\chi_{e^{+}}=\phi_{e^{+}}^{\max }-\tilde{\phi}=(2 n+1) \pi \pm G \Delta .
$$

This is in pretty good agreement with results of our full calculation shown in the left panel of Fig. 3 (recollect that in this calculation $\tilde{\phi}=0$ ). Thus, for pulse separation $G=4$ the choice of $n=0,-1$ leads to the bump position $\phi_{e^{+}}=$ $\pi+( \pm 2 \pi)$ and $-\pi+( \pm 2 \pi)$. This is coincides with results exhibited in the right panel of Fig. 3, since the phase factor
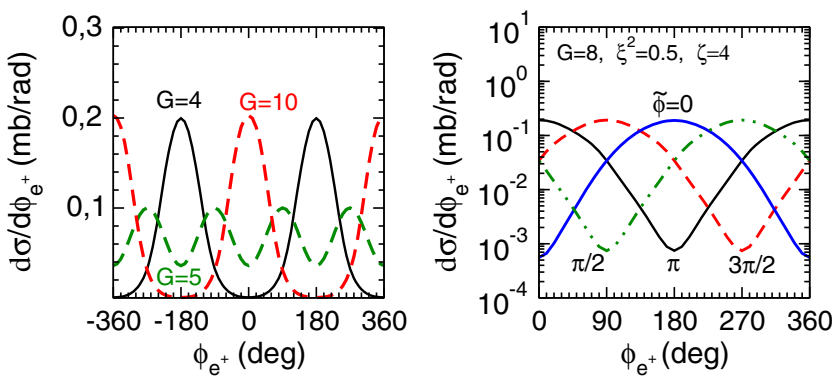

FIG. 3. Differential cross section $d \sigma / d \phi_{e^{+}}$of Eq. (3) for various values of the separation parameter $G$ and the carrier envelope phase $\tilde{\phi}$. For $N=1 / 2, \xi^{2}=0.5$ and $\zeta=4$. Left panel: $G=4$ (solid black curve), 5 (dashed green curve) and 10 (dashed red curve); $\tilde{\phi}=0$. Right panel: $G=8$ and $\tilde{\phi}=0$ (solid blue curve), $\pi / 2$ (dot-dashed green curve), $\pi$ solid black curve), $3 \pi / 2$ (dashed red curve). When plotting the curves as a function of $\chi_{e^{+}}=$ $\phi_{e^{+}}-\tilde{\phi}$ they coincide with the blue curve labeled by $\tilde{\phi}=0$. 
$( \pm 2 \pi)$ may be omitted. Similar results one can obtain for separation parameters $G=10$ with $\phi_{e^{+}}^{\max }=0, \pm 2 \pi$ and $G=5$ with $\phi_{e^{+}}^{\max }= \pm \pi / 2 \pm 3 \pi / 2$, respectively.

Let us now focus on the dependence of the azimuthal angular distribution on the separation parameter $G$, again for subcycle pulses with $N=1 / 2$. At $G=4 n$, the cross section of $e^{+} e^{-}$production is expected to be enhanced at

$$
\chi_{e^{+}}=(2 n+1) \pi
$$

The corresponding cross sections and anisotropies as a function of $\chi_{e^{+}}$calculated numerically using Eqs. (3) and (9), respectively, are displayed in the top and bottom of the left panels in Fig. 4. One can see sharp bumps in the cross sections and anisotropies at $\chi_{e^{+}}=\pi$, which coincide with our qualitative prediction (15).

The case $G=0$ is exceptional, since the two pulses completely overlap and the cross section is greatly enhanced. The main reason of such an enhancement is related to the modification of highly oscillating function $\Psi$ in Eq. (10). Now, the leading term reads

$$
\begin{gathered}
\Psi \simeq \ell \phi-2 z \Delta \cos (\Phi) \int_{-\infty}^{\frac{\phi}{\Delta}} d t f_{0}(t) \cos (t \Delta) \\
-2 z \Delta \sin (\Phi) \int_{-\infty}^{\frac{\phi}{\Delta}} d t f_{0}(t) \sin (t \Delta),
\end{gathered}
$$

which leads to the "renormalization" $z \rightarrow 2 z$ or $\xi^{2} \rightarrow 4 \xi^{2}$. The cross section scales with $\xi^{2}$ (see, e.g., [21] and the next
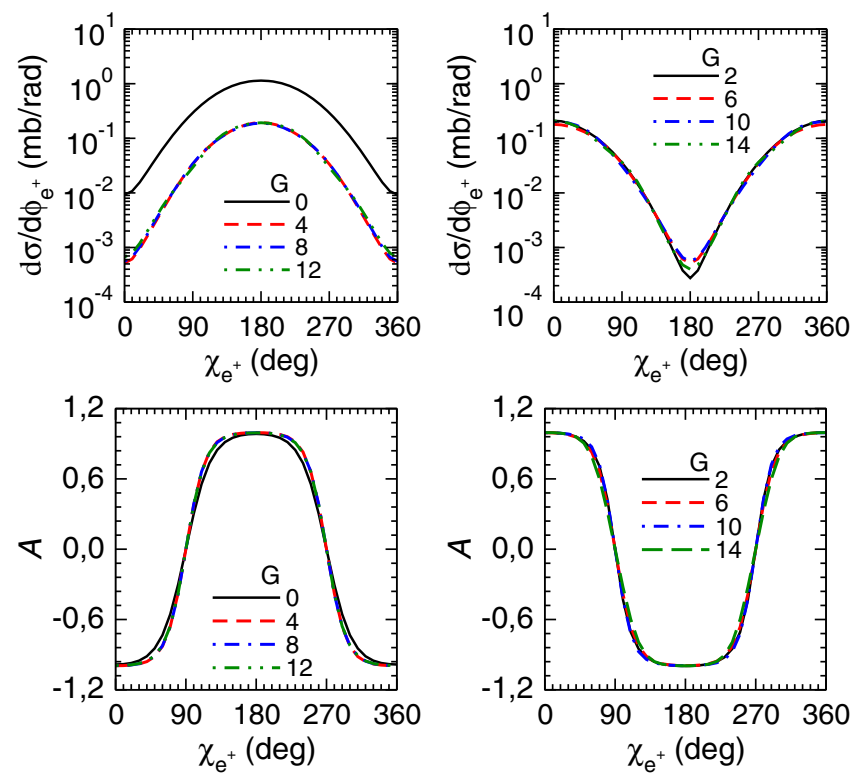

FIG. 4. Top panels: The differential cross section of Eq. (3) as a function of $\chi_{e^{+}}$for $N=1 / 2$, pulse separation parameters $G=0$, 4, 8, 12 and $G=2,6,10,14$ shown in the left and right panels, respectively. Bottom panels: The same as in the top panels, but for the anisotropy Eq. (9). subsection), therefore, one can see a strong enhancement of the cross section at $G=0$.

The situation changes dramatically for the separation parameter $G=2+4 n$. In this case, the cross sections and anisotropies are enhanced at $\phi_{e^{+}}-\tilde{\phi}=0$ and $2 \pi$ as illustrated in the right panels of Fig. 4. Here, we face also some (approximate) symmetry: the right column curves are generated by shifting $\chi_{e^{+}} \rightarrow \chi_{e^{+}} \pm \pi$.

Both examples show the explicit correlation between separation parameter $G$ and the carrier phase $\tilde{\phi}$ in the differential cross section $d \sigma / d \phi_{e^{+}}$, especially for $G<2$. For larger values of $G$, the cross section becomes independent of the separation parameter $G$.

A similar behavior is expected for wider pulses. For instance, in Fig. 5 we display results for the short pulse with $N=1$ corresponding to $\Delta=\pi$. Using Eq. (15) one can find that the bump-like structure with the bump position $\chi_{e^{+}}=\pi$ appears at $G=2 n$, as confirmed by our full numerical calculation exhibited in the left panels of Fig. 5. The values of the separation parameter $G=2 n+1$ result in an enhancement of the cross sections and anisotropies at $\chi_{e^{+}}=0$ and $2 \pi$ (cf. right panels of Fig. 5).

For wider pulses with $N \geq 2$ the bump structure and the nonmonotonic behavior become very weak. For example, Fig. 6 illustrates our result for the short pulses with $N=2$, that is $\Delta=2 \pi$. Nevertheless, some enhancement (weak bump structure at $\phi_{e^{+}}-\tilde{\phi}=\pi$ ) is expected at $G=0,1,2, \ldots$, while the enhancement at $\phi_{e}=0$ and $2 \pi$ is expected at half-integer values $G=1 / 2,3 / 2,5 / 2, \ldots$. Our corresponding result is depicted in Fig. 6. Our full numerical calculation does not support the qualitative prediction of (10).
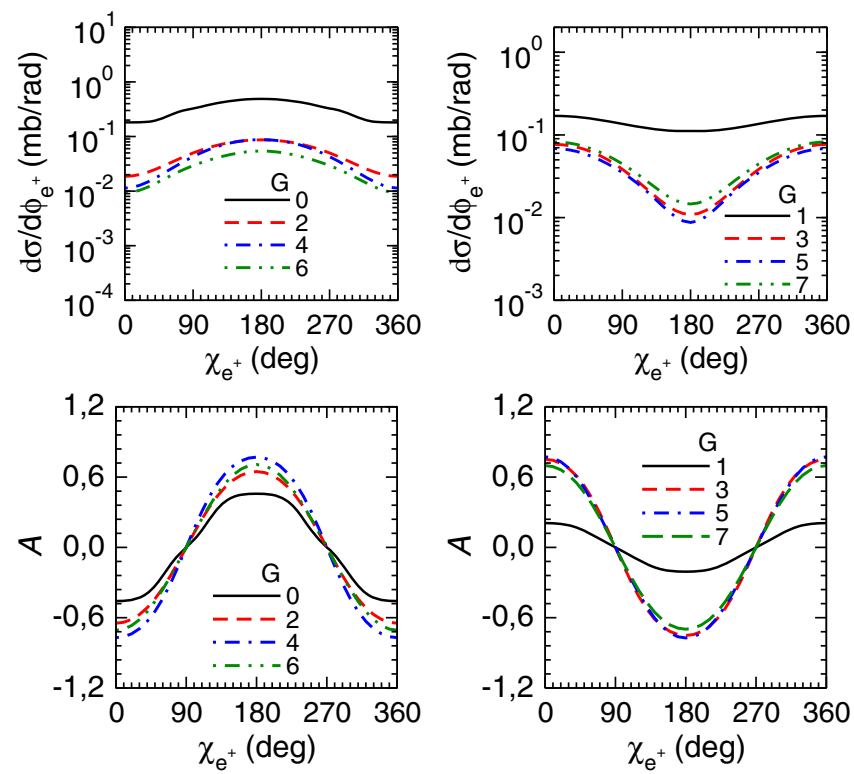

FIG. 5. The same as in Fig. 4 but for $N=1$ and pulse separation parameters $G=0,2,4,6$ and $G=1,3,5,7$ shown in the left and right panels, respectively. 

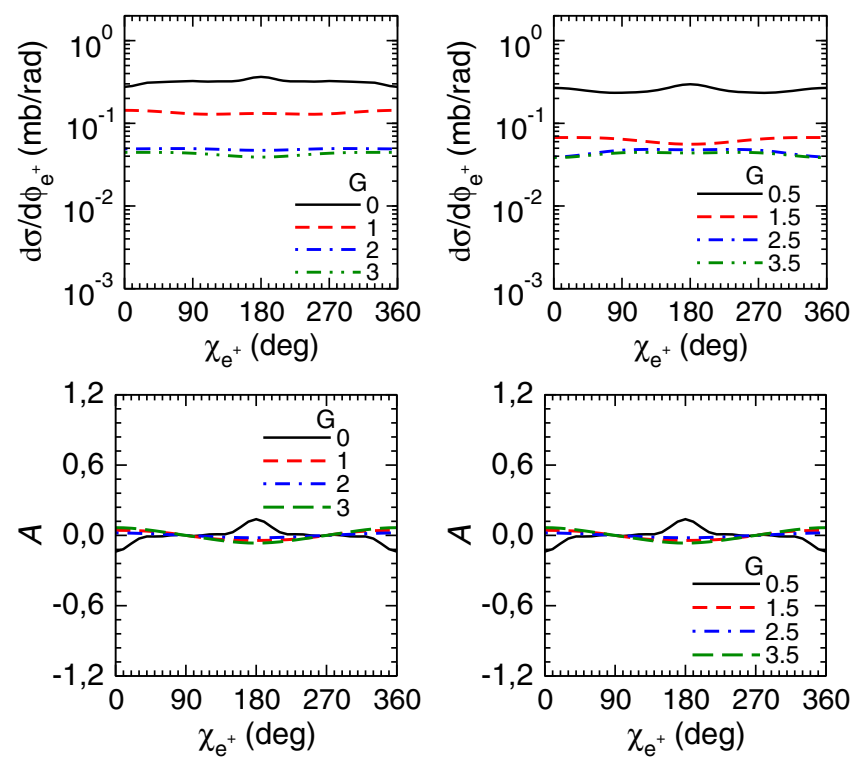

FIG. 6. The same as in Fig. 4 but for $N=2$ and separation parameters $G=0,1,2,3$ and $G=1 / 2,3 / 2,5 / 2,7 / 2$ shown in the left and right panels, respectively.

\section{B. Total cross section}

With the given parametrization $(1,2)$, the total cross section depends on the available energy $s$ (encoded in the subthreshold parameter $\zeta$ ), the laser intensity parameter $\xi$, the double-pulse distance parameter $G$, and the cycle number $N$ (encoded in the pulse width parameter $\Delta$ ): $\sigma\left(s ; \xi^{2}, G, N\right)$ since the CEP dependence disappears. A few of these dependencies are now considered. For simplicity and without loss of generality below we consider subcycle and short pulses with $N=1 / 2$ and $N=1$, respectively. Our analysis shows that wilder pulses do not bring qualitatively new results.

The dependence of the total cross section as a function of the field intensity encoded in $\xi^{2}$ is exhibited in Fig. 7. We choose a subthreshold pulse with $N=1 / 2$ and the separation parameter $G=4 n$ according to Fig. 4, left panels.
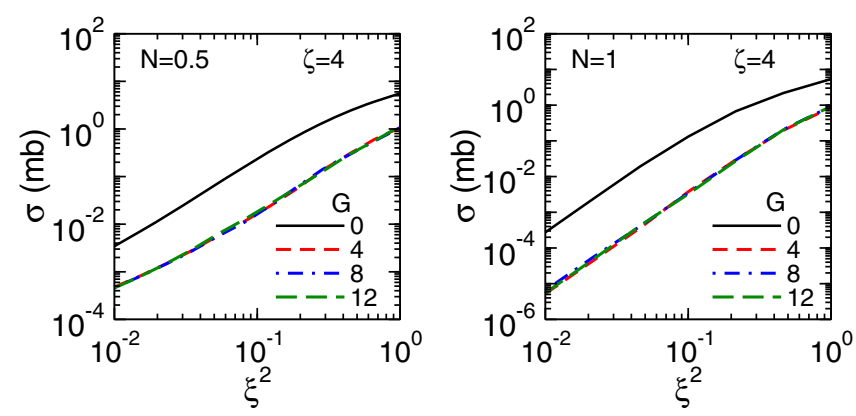

FIG. 7. The total cross section of the $e^{+} e^{-}$production as a function of the reduced field intensity parameter $\xi^{2}$. The results are for $N=1 / 2$, subthreshold parameter $\zeta=4$ and various values of the separation parameter $G$ listed in the legend.
One can see that all curves coincide with another at $G \geq 2$, where the dependence on the separation parameter disappears. Qualitatively, the behavior of the cross sections shown in the left and right panels are similar, being enhanced for subcycle pulse, especially for small vales of $\xi^{2}$.

This is correlated with results shown in Fig. 8, where the total cross sections as a function of the separation parameter $G$ for the subcycle $(N=1 / 2)$ and short $(N=1)$ pules are shown in the left and right panels, respectively. The calculations are for the subthreshold parameter $\zeta=5$ and the field intensities $\xi^{2}=0.1$ and 1 . The cross sections for $\xi^{2}=1$ are much greater compared to the case of $\xi^{2}=0.1$. One can see a significant enhancement of the cross sections at $G<2$ which is a consequence of strong overlap of the two pulses, discussed above, cf. top panels in Figs. 4 and 5. The cross sections at $G \geq 2$ are independent of $G$ and exhibit some plateau. For the subcycle pulses at chosen parameters within the accuracy of our calculation, the cross sections at the plateau are $\approx 1.4 \times 10^{-3} \mathrm{mb}$ and $\approx 0.32 \mathrm{mb}$ for $\xi^{2}=0.1$ and 1 , respectively. For the short pulses $(N=1)$, the result is qualitatively similar to that shown in the left panel but the cross sections are smaller compared to the case of $N=1 / 2$. Thus, the cross sections at plateau are $\approx 1.9 \times 10^{-4} \mathrm{mb}$ and $\approx 0.22 \mathrm{mb}$ for $\xi^{2}=0.1$ and 1 , respectively. The enhancement of the cross sections for the subcycle pulses compared to the short pulses, especially for small $\xi^{2}$, is consistent with our results exhibited in Fig. 7 and below in Fig. 9. Note that the results displayed in Figs. 7 and 8 are obtained at different values of the subthreshold parameter $\zeta=4$ and 5 , respectively.

The total cross section as a function of the subthreshold parameter $\zeta$ is exhibited in Fig. 9 for different values of the field intensity parameter $\xi^{2}$ and $G=4$. The left and right panels correspond to $N=1 / 2$ and $N=1$, respectively. One can see an almost exponential decrease of the cross section with $\zeta$. The slope of the curves decreases with increasing intensity of the electromagnetic field (or $\xi^{2}$ ). (Closer inspection shows that this result does not depend
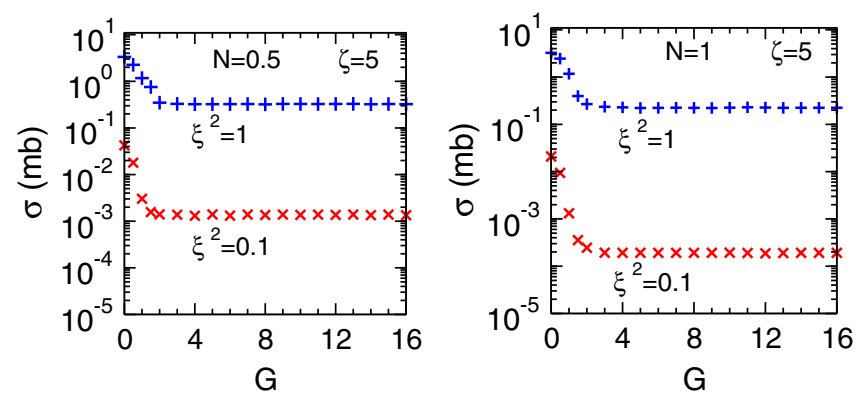

FIG. 8. The total cross section of the $e^{+} e^{-}$production as a function of separation parameter $G$ at $\xi^{2}=0.1$ and $\xi^{2}=1$, and $\zeta=5$. Left and right panels correspond to $N=1 / 2$ and $N=1$, respectively. 

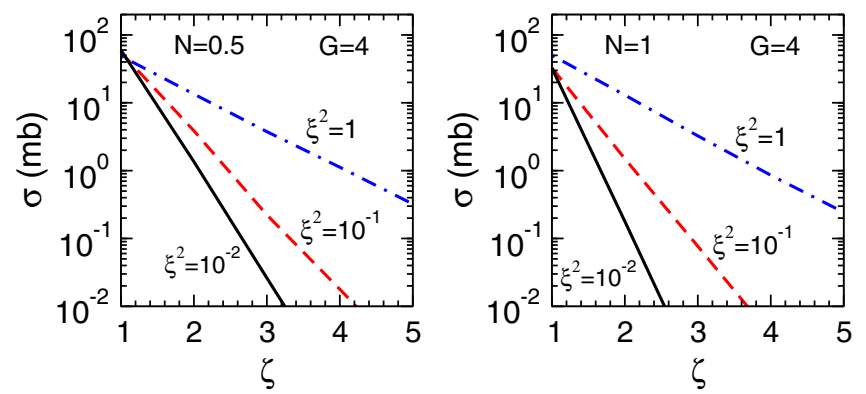

FIG. 9. The total cross section of the $e^{+} e^{-}$production as a function of subthreshold parameter $\zeta$ for various values of $\xi^{2}$ at fixed $G=4$ and $N=1 / 2$.

on the separation parameter $G \geq 2$ which is correlated with results exhibited in Fig. 8.) Qualitatively, results for subcycle and short pulses are similar. The curves in the case of subcycle pulses are slightly flatter.

\section{SUMMARY}

In summary we have analyzed the nonlinear (strongfield) Breit-Wheeler $e^{+} e^{-}$pair production in case of two consecutive circularly polarized short pulses with equal carrier phases and frequencies and strengths parameters. We find that for very short pulses with the number of oscillation in the pulse $N \leq 1$ the azimuthal angulardifferential cross section is sensitive to both the carrier phase $\tilde{\phi}$ and the phase separation parameter $G$. That is manifest in the bump-like behavior of the differential cross section. Results of the full numerical calculations coincide with simple analytical expressions. This means that the azimuthal angle distribution of outgoing Fermions may be used as a powerful method for determination of the structure of the short double pulses (or the carrier phase at fixed pulse geometry).

Nonidentical pulses are expected to lead to further interesting phenomena, which however we leave to separate work since a considerably extended parameter space is to be explored. The introduction of two different carrier envelope phases, for instance, will potentially diminish or even destroy the mentioned bump-like shapes of the cross sections and asymmetries. Staying with the minimumparameter double-pulse model we further note that the total cross section obeys a monotonic (near exponential) increase with the field intensity $\xi^{2}$ at fixed subthreshold parameter $\zeta$ and an exponential decrease of cross section with increasing $\zeta$ at fixed $\xi^{2}$. The total cross sections increases significantly for $G<2$ because of a strong interference in case of overlapping pulses. All these facts can be used in planning appropriate experiments.

\section{ACKNOWLEDGMENTS}

The authors gratefully acknowledge the collaboration with D. Seipt, T. Nousch, T. Heinzl, and useful discussions with A. Ilderton, K. Krajewska, M. Marklund, C. Müller, and R. Schützhold. We thank S. Glenzer for pointing out the content of Ref. [32] prior to publication. The work is supported by R. Sauerbrey and T. E. Cowan with respect to the study of fundamental QED processes for HIBEF.

\section{APPENDIX: EVALUATION OF THE PARTIAL PROBABILITY $w(\ell)$}

Here, for simplicity we choose the carrier phase $\tilde{\phi}$ equal to zero. Generalization for a finite $\tilde{\phi}$ is done in a straight forward manner. The starting point is (cf. [14,21,23])

$\frac{d \sigma}{d \phi_{e^{+}}}=\frac{\alpha^{2} \zeta}{8 m^{4} \xi^{2} N_{0}} \int_{\zeta}^{\infty} d \ell v \int_{-1}^{1} d \cos \theta_{e^{+}}\left|M_{f i}(\ell, u)\right|^{2}$,

where

$$
M_{f i}(\ell)=\sum_{i=0}^{3}\left[\bar{u}_{p^{\prime}} \hat{M}^{(i)} v_{p}\right] C^{(i)}(\ell),
$$

with

$$
\begin{aligned}
\hat{M}^{(0)} & =\not^{\prime \prime}, \quad \hat{M}^{(1)}=-\frac{e^{2} a^{2}\left(\varepsilon^{\prime} \cdot k\right) \not k}{2(k \cdot p)\left(k \cdot p^{\prime}\right)}, \\
\hat{M}^{(2,3)} & =\frac{e \not \phi_{(1,2)} \not \phi^{\prime \prime}}{2\left(k \cdot p^{\prime}\right)}-\frac{e \not^{\prime \prime} \not \phi_{(1,2)}}{2(k \cdot p)},
\end{aligned}
$$

where $u_{p^{\prime}}$ and $v_{p}$ are the Dirac spinors of the electron and positron, respectively, and $\varepsilon^{\prime}$ is the polarization four-vector of the probe photon $X$.

The functions $C^{(i)}(\ell)$ read

$$
\begin{aligned}
& C^{(0)}(\ell)=\frac{1}{2 \pi} \int_{-\infty}^{\infty} d \phi \mathrm{e}^{i \ell \phi-i \mathcal{P}(\phi)}, \\
& C^{(1)}(\ell)=\frac{1}{2 \pi} \int_{-\infty}^{\infty} d \phi f^{2}(\phi) \mathrm{e}^{i \ell \phi-i \mathcal{P}(\phi)}, \\
& C^{(2)}(\ell)=\frac{1}{2 \pi} \int_{-\infty}^{\infty} d \phi f(\phi) \cos \phi \mathrm{e}^{i \ell \phi-i \mathcal{P}(\phi)}, \\
& C^{(3)}(\ell)=\frac{1}{2 \pi} \int_{-\infty}^{\infty} d \phi f(\phi) \sin \phi \mathrm{e}^{i \ell \phi-i \mathcal{P}(\phi)},
\end{aligned}
$$

with the phase function $\mathcal{P}(\phi)$ from (8). The integrand of the function $C^{(0)}$ does not contain the envelope function $f(\phi)$ and therefore it is divergent. One can "regularize" it by using the prescription of [36] yielding

$$
\begin{aligned}
C^{(0)}(\ell)= & \frac{1}{2 \pi \ell} \int_{-\infty}^{\infty} d \phi\left[z \cos \left(\phi-\phi_{0}\right) f(\phi)-\xi^{2} \zeta u f^{2}(\phi)\right] \\
& \times \mathrm{e}^{i \ell \phi-i \mathcal{P}(\phi)}+\delta(\ell) \mathrm{e}^{-i \mathcal{P}(0)} .
\end{aligned}
$$

The divergence is isolated in the last term. However, it does not contribute because of kinematic considerations implying $\ell>0$. 
Utilizing Eqs. (A3)-(A5) and using the notation $Z_{i j}=$ $\frac{1}{4} \operatorname{Tr}\left[M^{(i)} M^{(j) \dagger}\right]$ one can express the mod-square of (A2) in the following form

$$
\begin{aligned}
& \frac{1}{4}\left|M_{f i}(\ell)\right|^{2} \\
&=\left|C^{(0)}\right|{ }^{2} Z_{00}+\left|C^{(1)}\right|{ }^{2} Z_{11}+\left|C^{(2)}\right|{ }^{2} Z_{22}+\left|C^{(3)}\right|^{2} Z_{33} \\
& \\
&++2 \operatorname{Re} C^{(0)} C^{(1) *} Z_{01}+2 \operatorname{Re} C^{(0)} C^{(2) *} Z_{02} \\
& \\
&+2 \operatorname{Re} C^{(0)} C^{(3) *} Z_{03}+2 \operatorname{Re} C^{(1)} C^{(2) *} Z_{12} \\
& \\
&+ 2 \operatorname{Re} C^{(1)} C^{(3) *} Z_{13}+2 \operatorname{Re} C^{(2)} C^{(3) *} Z_{23} .
\end{aligned}
$$

The averaging and sum over the spin variables in the initial and the final states is already executed. Trace calculations lead to

$$
\begin{aligned}
& Z_{00}=2\left(p \cdot p^{\prime}+2 m^{2}\right), \\
& Z_{22}=Z_{33}=\frac{\xi^{2} m^{2}\left((k \cdot p)^{2}+\left(k \cdot p^{\prime}\right)^{2}\right)}{(k \cdot p)\left(k \cdot p^{\prime}\right)}, \\
& Z_{01}=\xi^{2} m^{2}, \\
& Z_{02}=\frac{e\left(k \cdot p+k \cdot p^{\prime}\right)\left(\left(p^{\prime} \cdot a_{1}\right)(k \cdot p)-\left(p \cdot a_{1}\right)\left(k \cdot p^{\prime}\right)\right)}{(k \cdot p)\left(k \cdot p^{\prime}\right)}, \\
& Z_{03}=Z_{02}\left(a_{1} \rightarrow a_{2}\right), \\
& Z_{11}=Z_{12}=Z_{13}=Z_{23}=0
\end{aligned}
$$

which can be expressed through more convenient variables by

$$
\begin{aligned}
& Z_{00}=2 m^{2}\left(2 u_{\ell}+1\right), \\
& Z_{22}=Z_{33}=2 \xi^{2} m^{2}(2 u-1), \\
& Z_{01}=\xi^{2} m^{2}, \\
& Z_{02}=-2 m^{2} u_{\ell} \frac{z}{\ell} \cos \phi_{0}, \\
& Z_{03}=-2 m^{2} u_{\ell} \frac{z}{\ell} \sin \phi_{0}
\end{aligned}
$$

making Eq. (A6) surprisingly simple:

$$
\begin{aligned}
& \frac{1}{4}\left|M_{f i}(\ell)\right|^{2} \\
& =2 m^{2}\left(2 u_{0}+1\right)\left|C^{(0)}\right|^{2}+2 m^{2} \xi^{2}(2 u-1)\left(\left|C^{(2)}\right|^{2}+\left|C^{(3)}\right|^{2}\right) \\
& \quad+2 \xi^{2} m^{2} C^{(0)} C^{(1) *}-4 m^{2} u_{0} \frac{z}{\ell}\left(C^{(2)} \cos \phi_{0}\right. \\
& \left.\quad+C^{(3)} \sin \phi_{0}\right) C^{(0) *} .
\end{aligned}
$$

In principle, using the explicit expressions Eqs. (A4), (8) and (A5) for the functions $C^{(i)}$ one can evaluate $\frac{1}{4}\left|M_{f i}(\ell)\right|^{2}$ numerically. However, it turns out that it is more convenient to use another representation for the square of the matrix element, which allows us to carry out a qualitative analysis of the partial probability. For this aim we introduce new functions $Y_{\ell}(5)$ and $X_{\ell}$ (6) as well as $\tilde{Y}_{\ell}$ (7) which may be considered as the generalized Bessel functions for the finite e.m. pulse which allow to express the functions $C^{(i)}$ as

$$
\begin{aligned}
& C^{(0)}(\ell)=\tilde{Y}_{\ell}(z) \mathrm{e}^{i \ell \phi_{0}}, \\
& C^{(1)}(\ell)=X_{\ell}(z) \mathrm{e}^{i \ell \phi_{0}}, \\
& C^{(2)}(\ell)=\frac{1}{2}\left(Y_{\ell+1} \mathrm{e}^{i(\ell+1) \phi_{0}}+Y_{\ell-1} \mathrm{e}^{i(\ell-1) \phi_{0}}\right), \\
& C^{(3)}(\ell)=\frac{1}{2 i}\left(Y_{\ell+1} \mathrm{e}^{i(\ell+1) \phi_{0}}-Y_{\ell-1} \mathrm{e}^{i(\ell-1) \phi_{0}}\right) .
\end{aligned}
$$

The identity

$$
C^{(2)}(\ell) \cos \phi_{0}+C^{(3)}(\ell) \sin \phi_{0}=\frac{1}{2}\left(Y_{\ell+1}+Y_{\ell-1}\right) \mathrm{e}^{i \ell \phi_{0}}
$$

and Eq. (A10), together with some cumbersome algebra, lead to the final result (4) due to $w(\ell) \equiv \frac{1}{4 m^{2}}\left|M_{f i}(\ell)\right|^{2}$.
[1] G. A. Mourou, T. Tajima, and S. V. Bulanov, Optics in the relativistic regime, Rev. Mod. Phys. 78, 309 (2006).

[2] A. Di Piazza, C. Müller, K. Z. Hatsagortsyan, and C. H. Keitel, Extremely high-intensity laser interactions with fundamental quantum systems, Rev. Mod. Phys. 84, 1177 (2012).

[3] V. Yanovsky et al., Ultra-high intensity- 300-TW laser at $0.1 \mathrm{HZ}$ repetition rate, Opt. Express 16, 2109 (2008).

[4] http://www.clf.stfc.ac.uk/CLF/.

[5] http://www.eli-beams.eu.
[6] http://www.hiper-laser.org.

[7] https://www.ipfran.ru/english/science/las_phys.html.

[8] A. L. Cavalieri et al., Intense 1.5-cycle near infrared laser waveforms and their use for the generation of ultra-broadband soft-X-ray harmonic continua, New J. Phys. 9, 242 (2007).

[9] Zs. Major, S. Klingebiel, C. Skrobol, I. Ahmad, C. Wandt, S. A. Trushin, F. Krausz, and S. Karsch, Status of the Petawatt Field Synthesizer-pump-seed synchronization measurements, AIP Conf. Proc. 1228, 117 (2010). 
[10] F. Mackenroth and A. Di Piazza, Nonlinear Compton scattering in ultra-short laser pulses, Phys. Rev. A 83, 032106 (2011).

[11] X. Feng, S. Gilbertson, H. Mashiko, He Wang, S. D. Khan, M. Chini, Y. Wu, K. Zhao, and Z. Chang, Generation of Isolated Attosecond Pulses with 20 to 28 Femtosecond Lasers, Phys. Rev. Lett. 103, 183901 (2009).

[12] F. Krausz and M. Ivanov, Attosecond physics, Rev. Mod. Phys. 81, 163 (2009).

[13] M. J. A. Jansen and C. Müller, Strong-field Breit-Wheeler pair production in short laser pulses: Identifying multiphoton interference and carrier-envelope phase effects, Phys. Rev. D 93, 053011 (2016).

[14] A. I. Titov, B. Kämpfer, A. Hosaka, T. Nousch, and D. Seipt, Determination of the carrier envelope phase for short, circularly polarized laser pulses, Phys. Rev. D 93, 045010 (2016).

[15] J. X. Li, Y. Y. Chen, K. Z. Hatsagortsyan, and C. H. Keitel, Single-Shot Carrier-Envelope Phase Determination of Long Superintense Laser Pulses, Phys. Rev. Lett. 120, 124803 (2018).

[16] M. J. A. Jansen and C. Müller, Strong-field Breit-Wheeler pair production in two consecutive laser pulses with variable time delay, Phys. Lett. B 766, 71 (2017).

[17] T. G. Blackburn and M. Marklund, Nonlinear Breit-Wheeler pair creation with bremsstrahlung $\gamma$ rays, Plasma Phys. Controlled Fusion 60, 054009 (2018).

[18] T. G. Blackburn, A. Ilderton, C. D. Murphy, and M. Marklund, Scaling laws for positron production in laserelectron-beam collisions, Phys. Rev. A 96, 022128 (2017).

[19] V. I. Ritus, Quantum effects of the interaction of elementary particles with an intense electromagnetic field, J. Sov. Laser Res. (United States), 6, 497 (1985).

[20] K. Krajewska and J. Z. Kaminski, Breit-Wheeler process in intense short laser pulses, Phys. Rev. A 86, 052104 (2012).

[21] A. I. Titov, B. Kämpfer, A. Hosaka, and H. Takabe, Quantum processes in short and intensive electromagnetic fields, Phys. Part. Nucl. 47, 456 (2016).

[22] A. I. Titov, B. Kämpfer, H. Takabe, and A. Hosaka, BreitWheeler process in very short electromagnetic pulses, Phys. Rev. A 87, 042106 (2013).

[23] A. I. Titov, H. Takabe, B. Kämpfer, and A. Hosaka, Enhanced Subthreshold $e^{+} e^{-}$Production in Short Laser Pulses, Phys. Rev. Lett. 108, 240406 (2012).
[24] T. Nousch, D. Seipt, B. Kämpfer, and A. I. Titov, Pair production in short laser pulses near threshold, Phys. Lett. B 715, 246 (2012).

[25] M. J. A. Jansen, J. Z. Kamiski, K. Krajewska, and C. Müller, Strong-field Breit-Wheeler pair production in short laser pulses: Relevance of spin effects, Phys. Rev. D 94, 013010 (2016).

[26] M. J. A. Jansen and C. Müller, Strongly enhanced pair production in combined high- and low-frequency laser fields, Phys. Rev. A 88, 052125 (2013).

[27] T. Nousch, D. Seipt, B. Kämpfer, and A. I. Titov, Spectral caustics in laser assisted Breit-Wheeler process, Phys. Lett. B 755, 162 (2016).

[28] A. Otto, T. Nousch, D. Seipt, B. Kämpfer, D. Blaschke, A. D. Panferov, S. A. Smolyansky, and A. I. Titov, Pair production by Schwinger and Breit-Wheeler processes in bi-frequent fields, J. Plasma Phys. 82, 655820301 (2016).

[29] A. Di Piazza, First-order strong-field QED processes in a tightly focused laser beam, Phys. Rev. A 95, 032121 (2017).

[30] A. Di Piazza, Nonlinear Breit-Wheeler Pair Production in a Tightly Focused Laser Beam, Phys. Rev. Lett. 117, 213201 (2016).

[31] K. Krajewska and J.Z. Kamiski, Coherent combs of antimatter from nonlinear electron-positron-pair creation, Phys. Rev. A 90, 052108 (2014).

[32] F.-J. Decker, K. L. F. Bane, R. N. Coffee, W. S. Colocho, S. Gilevich, S. H. Glenzer, A. A. Lutman, A. Miahnahri, D. F. Ratner, J. C. Sheppard, and S. Vetter, Two and multiple bunches at LCLS (JACoW Publishing, Geneva, Switzerland, 2018).

[33] F. Mackenroth, N. Kumar, N. Neitz, and C. H. Keitel, Nonlinear Compton Scattering of an Ultra-intense Laser Pulse in a Plasma, arXiv:1805.01762 [Phys. Rev. Lett. (to be published)].

[34] B. Kämpfer and O.P. Pavlenko, On the Ter-Mikaelian and Landau-Pomeranchuk effects for induced soft gluon radiation in a QCD medium, Phys. Lett. B 477, 171 (2000).

[35] A. I. Titov, B. Kämpfer, T. Shibata, A. Hosaka, and H. Takabe, Laser pulse-shape dependence of Compton scattering, Eur. Phys. J. D 68, 299 (2014).

[36] M. Boca and V. Florescu, Nonlinear Compton scattering with a laser pulse, Phys. Rev. A 80, 053403 (2009). 